\title{
STEPS TOWARD DETERMINATION OF THE SIZE AND STRUCTURE OF THE BROAD-LINE REGION IN ACTIVE GALACTIC NUCLEI. VI. VARIABILITY OF NGC 3783 FROM GROUND-BASED DATA ${ }^{1}$
}

G. M. Stirpe ${ }^{2}$ C. Winge, ${ }^{3,4}$ B. Altieri, ${ }^{5,6}$ D. Alloin, ${ }^{7}$ E. L. Aguero, ${ }^{8,9}$ G. C. Anupama,${ }^{10}$ R. Ashley, ${ }^{11}$ R. Bertram, ${ }^{12,13}$ J. H. Calderon, ${ }^{8}$ R. M. Catchpole, ${ }^{14}$ R. L. M. Corradi, ${ }^{5,15}$ E. Covino, ${ }^{16}$ H. A. DotTori, ${ }^{3}$ M. W. Feast, ${ }^{11}$ K. K. Ghosh, ${ }^{17}$ R. Gil Hutton, ${ }^{18,19}$ I. S. Glass, ${ }^{20}$ E. K. Grebel, ${ }^{5}$ L. Jorda, ${ }^{5,7}$ C. Koen, ${ }^{20}$ C. D. Laney, ${ }^{20}$ M. Maia, ${ }^{21}$ F. Marang, ${ }^{20}$ Y. D. Mayya, ${ }^{22}$ N. Morrell, ${ }^{23}$ Y. NaKada, ${ }^{24}$ M. G. Pastoriza, ${ }^{3}$ A. K. Pati, ${ }^{22}$ D. Pelat, ${ }^{7}$ B. M. Peterson, ${ }^{12}$ T. P. Prabhu, ${ }^{22}$ G. Roberts, ${ }^{20}$ R. Sagar, ${ }^{22}$ I. Salamanca, ${ }^{7}$ K. Sekiguchi, ${ }^{20}$ T. Storchi-Bergmann, ${ }^{3}$ A. Subramaniam, ${ }^{22}$ H. Van Winckel,${ }^{5,25}$ F. van WyK, ${ }^{20}$ M. Villada, ${ }^{8,9}$ R. M. Wagner, ${ }^{12,13}$ P. A. Whitelock, ${ }^{20}$ H. Winkler, ${ }^{26}$ J. Clavel, ${ }^{6}$ M. DietriCh, ${ }^{27}$ W. Kollatschny, ${ }^{27}$ P. T. O’Brien, ${ }^{28}$ G. C. Perola,${ }^{29}$ M. C. Recondo-González, ${ }^{30}$

P. Rodríguez-Pascual, ${ }^{30}$ and M. Santos-Lleó ${ }^{7}$

Received 1993 June 18; accepted 1993 October 25

\begin{abstract}
The Seyfert 1 galaxy NGC 3783 was intensely monitored in several bands between 1991 December and 1992 August. This paper presents the results from the ground-based observations in the optical and near-IR bands, which complement the data set formed by the International Ultraviolet Explorer (IUE) spectra, discussed elsewhere. Spectroscopic and photometric data from several observatories were combined in order to obtain well-sampled light curves of the continuum and of $\mathrm{H} \beta$. During the campaign the source underwent significant variability. The light curves of the optical continuum and of $\mathrm{H} \beta$ display strong similarities to those obtained with the IUE. The near-IR flux did not vary significantly except for a slight increase at the end of the campaign.

The cross-correlation analysis shows that the variations of the optical continuum have a lag of 1 day or less with respect to those of the UV continuum, with an uncertainty of $\leq 4$ days. The integrated flux of $\mathrm{H} \beta$ varies with a delay of about 8 days. These results confirm that (1) the continuum variations occur simultaneously or with a very small lag across the entire UV-optical range, as in the Seyfert galaxy NGC 5548; and (2) the emission lines of NGC 3783 respond to ionizing continuum variations with less delay than those of NGC 5548. As observed in NGC 5548, the lag of $\mathrm{H} \beta$ with respect to the continuum is greater than those of the high-ionization lines.
\end{abstract}

Subject headings: galaxies: active - galaxies: individual (NGC 3783) - galaxies: nuclei galaxies: photometry — galaxies: Seyfert

\footnotetext{
${ }^{1}$ Partly based on observations collected at the European Southern Observatory, La Silla, Chile.

2 Osservatorio Astronomico di Bologna, Via Zamboni 33, 40126 Bologna, Italy; stirpe@alma02.bo.astro.it.

${ }^{3}$ Departamento de Astronomia, Instituto de Física, Universidade Federal do Rio Grande do Sul, Avenida Bento Gonçalves, 9500, CP15051, CEP 91500 , Porto Alegre, RS, Brazil.

${ }^{4}$ Visiting Astronomer at the Cerro Tololo Inter-American Observatory of the National Optical Observatories, operated by AURA, Inc., under contract with the National Science Foundation.

${ }^{5}$ European Southern Observatory, Casilla 19001, Santiago 19, Chile.

6 ISO Observatory, ESA Astrophysics Division, ESTEC, Postbus 299, 2200 AG Noordwijk, The Netherlands.

${ }^{7}$ Observatoire de Paris, URA173 CNRS, Université Paris 7, Place Jules Janssen, 92195, Meudon Principal Cedex, France.

${ }^{8}$ Observatorio Astronómico de Córdoba, Córdoba, Argentina.

9 Visiting Astronomer at the Complejo Astronómico El Leoncito (CASLEO), San Juan, Argentina.

${ }^{10}$ Inter-University Centre for Astronomy and Astrophysics, Post Bag 4, Ganeshkhind, Pune 411007, India.

${ }^{11}$ Department of Astronomy, University of Cape Town, Private Bag, 7700 Rondebosch, Cape Town, South Africa.

12 Department of Astronomy, The Ohio State University, 174 West 18th Avenue, Columbus, $\mathrm{OH} 43210$.

${ }^{13}$ Postal address: Lowell Observatory, Mars Hill Road, 1400 West, Flagstaff, AZ 86001 .
}

${ }^{14}$ Royal Greenwich Observatory, Madingley Road, Cambridge CB3 0HA, UK

15 Dipartimento di Astronomia, Università di Padova, Vicolo dell'Osservatorio 5, 35122 Padova, Italy.

16 Osservatorio Astronomico di Capodimonte, Via Moiariello 16, 80131 Napoli, Italy.

${ }_{17}$ Vainu Bappu Observatory, Indian Institute of Astrophysics, Kavalur, Alangayam 635701, Tamil Nadu, India.

${ }^{18}$ Observatorio Astronómico Felix Aguilar, San Juan, Argentina.

19 Yale Southern Observatory, San Juan, Argentina.

${ }^{20}$ South African Astronomical Observatory, P.O. Box 9, Observatory 7935, Cape Town, South Africa.

21 Observatorio Nacional, CNPq, Rio de Janeiro, Brazil.

22 Indian Institute of Astrophysics, Bangalore 560 034, India.

${ }^{23}$ Observatorio Astronómico de La Plata, La Plata, Argentina.

${ }^{24}$ Kiso Observatory, Institute of Astronomy, University of Tokyo, Japan.

${ }^{25}$ Astronomisch Instituut, Katholieke Universiteit Leuven, Celestijnenlaan 200 B, 3001 Heverlee, Belgium.

${ }^{26}$ Department of Physics, Vista University, Soweto Campus, Private Bag X09, 2013 Bertsham, Johannesburg, South Africa.

${ }^{27}$ Universitäts-Sternwarte, Geismarlandstra $\beta$ e 11, W-3400 Göttingen, Germany.

${ }^{28}$ Department of Physics and Astronomy, UCL, Gower Street, London WC1E 6BT, UK.

${ }^{29}$ Istituto Astronomico dell'Università, Via Lancisi 29, 00161 Roma, Italy.

${ }^{30}$ ESA IUE Observatory, P.O. Box 50727, 28080 Madrid, Spain. 


\section{INTRODUCTION}

The nucleus of the Seyfert 1 galaxy NGC $3783\left(\alpha_{1950.0}=\right.$ $11^{\mathrm{h}} 36^{\mathrm{m}} 33^{\mathrm{s}} .0, \delta_{1950.0}=37^{\circ} 27^{\prime} 41^{\prime \prime} ; z=0.0097 ; m_{V} \sim 13$ ) is one of the brightest and therefore best-studied active galactic nuclei (AGNs) of the southern hemisphere. The optical spectrum of this source, which has been extensively studied by Pelat, Alloin, \& Fosbury (1981) and Evans (1988), presents very strong emission lines, with a high degree of ionization (Fig. 1). Variations of the broad components of the optical emission lines have been reported by Menzies \& Feast (1983), Stirpe, de Bruyn, \& van Groningen (1988), Evans (1989), Winge, Pastoriza, \& Storchi-Bergmann (1990), Winge et al. (1992), and Winkler (1992). De Ruiter \& Lub (1986) and Winkler et al. (1992) present multiband photometric observations, which show that the source has varied by several tenths of a magnitude. Photometric $U$ data shown by Glass (1992) display variations in excess of $1.1 \mathrm{mag}$. Variations have also been observed in the near-IR by Glass (1992), from $\sim 0.5 \mathrm{mag}$ at $J(1.25 \mu \mathrm{m})$ to $\sim 1.1 \mathrm{mag}$ at $L(3.5 \mu \mathrm{m})$.

Because of this history of variability, NGC 3783 was judged a suitable candidate for an intensive monitoring campaign, similar to that carried out on NGC 5548 by the International AGN Watch consortium (Clavel et al. 1991, Peterson et al. 1991, 1992, Dietrich et al. 1993, and Peterson et al. 1994, hereafter Papers I-IV and VII, respectively; Maoz et al. 1993; see also the review by Peterson 1993). The monitoring of NGC 3783 took place between 1991 December and 1992 August, and made use of the International Ultraviolet Explorer (IUE) and of several ground-based telescopes, mainly in the southern hemisphere. The results of the IUE observations are presented by Reichert et al. (1994, hereafter Paper V). This paper presents the first results from the ground-based spectroscopic and photometric observations in the optical and near-IR bands. Section 2 gives a description of the observations, $\S 3$ describes how the spectra were corrected for slit losses, $\S \S 4$ and 5 describe how the light curves of the continuum and of $\mathrm{H} \beta$ were obtained, $\S 6$ presents a discussion and analysis of the light curves, and $\S 7$ summarizes our main conclusions.

\section{OBSERVATIONS}

\subsection{Spectroscopy}

Spectroscopic observations were obtained at Cerro Tololo Inter-American Observatory (CTIO) in Chile; at the European Southern Observatory (ESO), La Silla, Chile; at the Complejo Astronómico El Leoncito (CASLEO), Argentina; at the Ohio State University (OSU) Perkins reflector at Lowell Obsevatory; and at Vainu Bappu Observatory, Kavalur, India. All spectra were recorded on CCDs, with the exception of those taken at the CTIO $1 \mathrm{~m}$ telescope, where the 2D-FRUTTI detector was used. The bulk of the data comes from this telescope and from the ESO $1.5 \mathrm{~m}$ telescope: the CTIO spectra were all obtained with the same instrumental setup, and therefore form a very homogeneous data set; the ESO spectra vary widely in resolution and wavelength coverage, because the program shared nights with other scheduled observers, who had priority in the choice of the grating. A journal of the observations is given in Table 1: the columns list respectively the Julian Date minus 2,440,000 and the UT date at the midpoint of the integration, a code $(\mathrm{A}-\mathrm{E})$ which indicates where the spectrum was obtained, the air mass at the midpoint of the integration, the area of the aperture (slit width $\times$ length) in square arcseconds, the resolution and wavelength range in angstroms, and the integration times in seconds. The seeing was generally between $1^{\prime \prime}$ and $2^{\prime \prime}$. Epochs with subarcsecond seeing include JD 2,448,609, JD 2,448,649, and JD 2,448,824. Bad seeing $\left(\sim 3^{\prime \prime}\right)$ was recorded on JD 2,448,820.

All spectra were reduced using standard techniques. This paper presents the light curve of the optical continuum and $\mathrm{H} \beta$ only; the analysis of other emission lines will be presented in a future paper. Unless otherwise stated, all wavelengths quoted in this paper are in the observed frame.

\subsection{Optical Photometry}

Photoelectric $U B V(R I)_{C}$ photometry was obtained at the $0.5 \mathrm{~m}$ telescope of the South African Astronomical Observatory at Sutherland, using the SAAO photometer equipped with a Hamamatsu GaAs photomultiplier tube. An aperture of $20^{\prime \prime}$ in diameter was used. The seeing varied between 1 " and $5^{\prime \prime}$. The observations and reduction were performed as described in Winkler et al. (1992). The results are listed in Table 2. The uncertainties on the magnitudes are $0.03 \mathrm{mag}$.

Photoelectric $U B V$ photometry was obtained at the $0.7 \mathrm{~m}$ Charles D. Perrin telescope at the Observatorio Astronómico Felix Aguilar (OAFA), San Juan, Argentina, through an aperture of $33^{\prime \prime}$ in diameter. The photometer was equipped with an RCA 31034A photomultiplier tube. The seeing was between $2^{\prime \prime}$ and $3^{\prime \prime}$ at all epochs. The data were calibrated using standard star fluxes from Landolt (1973, 1983). The measurements are listed in Table 3. The first part of the campaign was affected by the presence in the atmosphere of dust from the Hudson volcano, which caused some color measurements to be unreliable: these have been omitted from the table. The uncertainties on the magnitudes are $0.03 \mathrm{mag}$.

CCD photometry in $B V R$ was obtained at the $1 \mathrm{~m}$ telescope of the Vainu Bappu Observatory (VBO). The seeing was between $2^{\prime \prime}$ and $3^{\prime \prime}$ for all observations. Bias subtraction and flat-fielding was performed on the frames, using Starlink routines. The flux of NGC 3783 was measured with circular apertures with a diameter of $18^{\prime \prime}$, and calibrated with the nearby star SAO 202668, exposed on the same frames. The results are listed in Table 4. The uncertainties are $0.05 \mathrm{mag}$ for the magnitudes, and 0.03 mag for the colors.

There is a systematic difference between $B-V$ values obtained at SAAO and VBO on close dates, which cannot be accounted for entirely by the slightly different apertures used. Given the peculiar shape of AGN spectra, it is likely that the difference is caused by the large color term required to convert the observed VBO values to the Johnson photometric system. The accuracies quoted do not take this systematic effect into account, but are correct within the individual data sets.

\subsection{Infrared Photometry}

$J H K L$ photometry was obtained at SAAO, through a $12^{\prime \prime}$ aperture, using the Mk III infrared photometer attached to the $1.9 \mathrm{~m}$ telescope. The observations and reduction took place as described by Glass (1992). The chopping distance was 30" north and south of the nucleus. Tests with larger chopping distances were made on NGC 3783 in 1988 March: a distance of $60^{\prime \prime}$ yielded fluxes which differed by at most $0.01 \mathrm{mag}$ from those obtained with the $30^{\prime \prime}$ distance. Therefore, the latter was considered sufficient.

The fluxes were calibrated in the SAAO standard system (Carter 1990), using the standard star HR 4523. Other standards observed during the same nights provided a check on the photometric accuracy. The magnitudes are given in Table 5. 
TABLE 1

JOURNAL OF SPECTROSCOPIC OBSERVATIONS

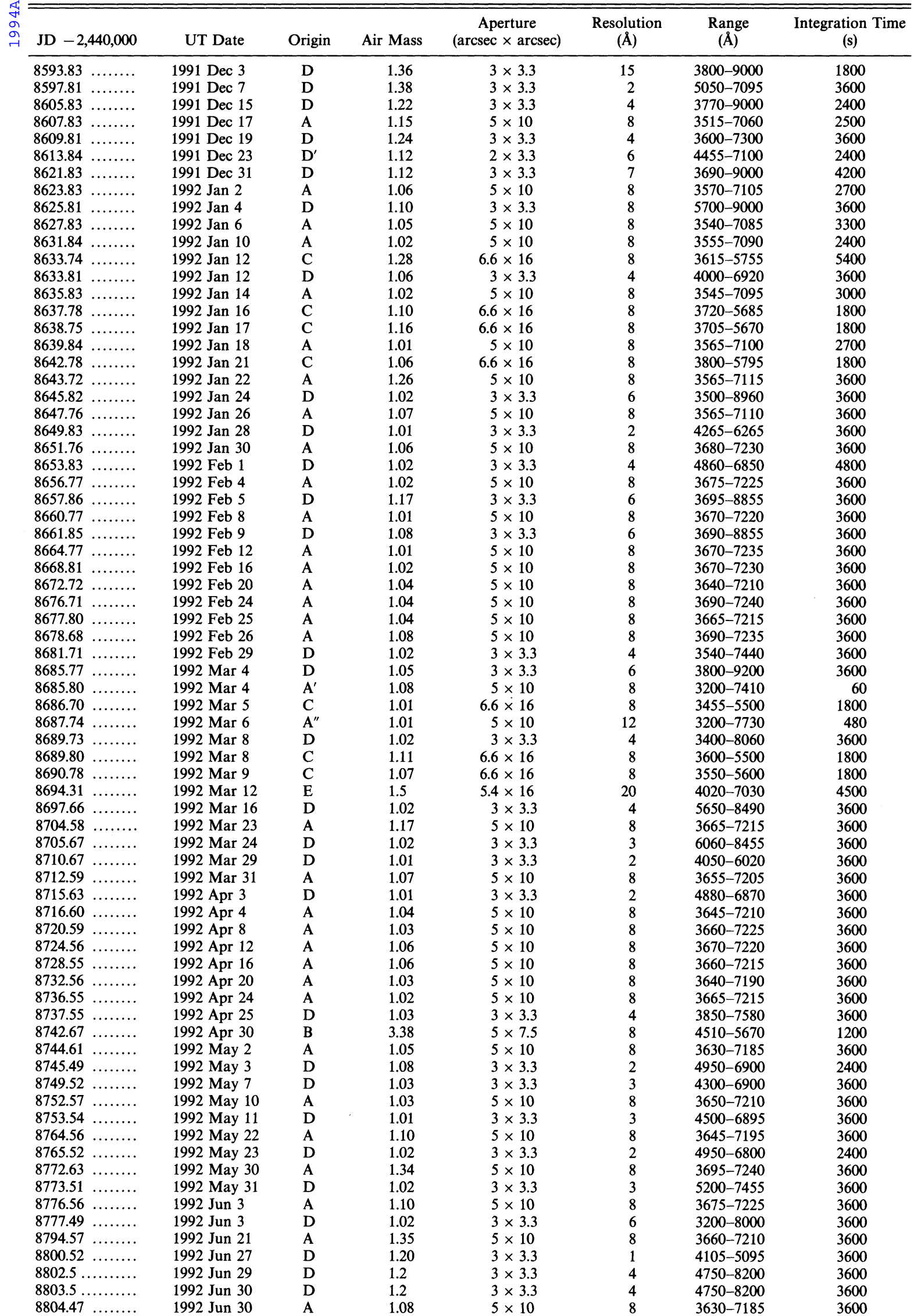

(C) American Astronomical Society - Provided by the NASA Astrophysics Data System 
TABLE $1-$ Continued

\begin{tabular}{|c|c|c|c|c|c|c|c|}
\hline JD $-2,440,000$ & UT Date & Origin & Air Mass & $\begin{array}{c}\text { Aperture } \\
(\operatorname{arcsec} \times \text { arcsec) }\end{array}$ & $\begin{array}{c}\text { Resolution } \\
(\AA)\end{array}$ & $\begin{array}{c}\text { Range } \\
(\AA)\end{array}$ & $\begin{array}{l}\text { Integration Time } \\
\text { (s) }\end{array}$ \\
\hline 8805.5 & 1992 Jul 2 & D & 1.2 & $3 \times 3.3$ & 15 & $3400-8400$ & 3600 \\
\hline 8806.57 & 1992 Jul 3 & A & 1.58 & $5 \times 10$ & 8 & $3600-7150$ & 3600 \\
\hline 8808.47 . & $1992 \mathrm{Jul} 4$ & A & 1.10 & $5 \times 10$ & 8 & $3590-7140$ & 3600 \\
\hline $8820.55 \ldots \ldots \ldots$ & 1992 Jul 17 & D & 1.96 & $3 \times 3.3$ & 4 & $3700-7000$ & 1200 \\
\hline $8822.48 \ldots \ldots \ldots$ & 1992 Jul 18 & A & 1.25 & $5 \times 10$ & 8 & $3590-7145$ & 5400 \\
\hline $8822.53 \ldots \ldots \ldots$ & 1992 Jul 19 & D & 1.61 & $3 \times 3.3$ & 4 & $3700-7000$ & 4500 \\
\hline $8823.50 \ldots \ldots \ldots$ & 1992 Jul 20 & D & $>2$ & $3 \times 3.3$ & 4 & $3700-7000$ & 3600 \\
\hline $8824.47 \ldots \ldots \ldots$ & 1992 Jul 20 & $\bar{A}$ & 1.23 & $5 \times 10$ & 8 & $3590-7140$ & 5400 \\
\hline $8824.52 \quad \ldots \ldots \ldots$ & 1992 Jul 21 & D & 1.6 & $3 \times 3.3$ & 4 & $3700-7000$ & 1800 \\
\hline $8826.47 \quad \ldots \ldots \ldots$ & 1992 Jul 22 & $\bar{A}$ & 1.25 & $5 \times 10$ & 8 & $3590-7140$ & 1800 \\
\hline $8830.48 \ldots \ldots \ldots$ & 1992 Jul 26 & A & 1.31 & $5 \times 10$ & 8 & $3645-7195$ & 5400 \\
\hline $8832.46 \ldots \ldots \ldots$ & 1992 Jul 28 & A & 1.32 & $5 \times 10$ & 8 & $3645-7195$ & 5400 \\
\hline $8841.49 \quad \ldots \ldots \ldots$ & 1992 Aug 6 & D & 1.76 & $3 \times 3.3$ & 6 & $4600-8000$ & 3600 \\
\hline $8844.50 \ldots \ldots \ldots$ & 1992 Aug 9 & D & 1.83 & $3 \times 3.3$ & 6 & $4600-8200$ & 3600 \\
\hline
\end{tabular}

Note.-Codes for data origin: A-1.0 m CTIO telescope + spectrograph + 2D FRUTTI; A'-4.0 m CTIO telescope + RitcheyChrétien spectrograph + CCD; $\mathrm{A}^{\prime \prime}-1.5 \mathrm{~m}$ CTIO telescope + spectrograph + CCD; B-1.8 m Perkins telescope + Ohio State spectrograph + CCD; C-2.2 m CASLEO telescope + Boller \& Chivens spectrograph + CCD; D-1.5 m ESO spectroscopic telescope + Boller \& Chivens spectrograph + CCD; D'-2.2 m ESO/MPI telescope + EFOSC2 + CCD; E-1.0 m VBO telescope + Zeiss spectrograph + CCD

TABLE 2

Photóilectric Photometry from SAAO (20" aperture)

\begin{tabular}{|c|c|c|c|c|c|}
\hline JD $-2,440,000$ & $V$ & $B-V$ & $U-B$ & $V-R_{\mathrm{C}}$ & $V-I_{\mathrm{C}}$ \\
\hline 8610.56 . & 12.93 & 0.63 & -0.67 & 0.53 & 1.00 \\
\hline 8622.55 . & 12.96 & 0.61 & -0.65 & 0.56 & 1.02 \\
\hline $8630.57 .$. & 12.96 & 0.66 & -0.60 & 0.55 & 1.04 \\
\hline $8634.55 \ldots$ & 12.98 & 0.65 & -0.60 & 0.56 & 1.03 \\
\hline $8638.57 \ldots$ & 12.96 & 0.65 & -0.63 & 0.57 & 1.04 \\
\hline $8644.56 \ldots$ & 12.94 & 0.60 & -0.65 & 0.56 & 1.02 \\
\hline $8651.58 \ldots$ & 12.94 & 0.63 & -0.66 & 0.55 & 1.02 \\
\hline $8655.58 \ldots$ & 12.93 & 0.60 & -0.68 & 0.55 & 1.03 \\
\hline 8659.54 . & 12.92 & 0.60 & -0.68 & 0.55 & 1.01 \\
\hline $8663.53 .$. & 12.93 & 0.62 & -0.66 & 0.57 & 1.04 \\
\hline $8671.52 .$. & 12.98 & 0.61 & -0.59 & 0.56 & 1.03 \\
\hline 8684.52 . & 13.00 & 0.67 & -0.60 & 0.57 & 1.05 \\
\hline $8688.60 \ldots$ & 13.11 & 0.68 & -0.58 & 0.58 & 1.07 \\
\hline $8700.45 .$. & 13.05 & 0.72 & -0.55 & 0.58 & 1.06 \\
\hline $8704.42 .$. & 13.08 & 0.70 & -0.57 & 0.58 & 1.09 \\
\hline $8714.41 \ldots$ & 13.03 & 0.63 & -0.65 & 0.55 & 1.02 \\
\hline $8717.50 \ldots$ & 12.99 & 0.64 & -0.63 & 0.54 & 1.03 \\
\hline $8729.38 \ldots \ldots \ldots$ & 12.97 & 0.63 & -0.65 & 0.56 & 1.04 \\
\hline $8733.36 \ldots$ & 12.97 & 0.63 & -0.64 & 0.56 & 1.02 \\
\hline $8743.40 \ldots \ldots \ldots$ & 12.96 & 0.65 & -0.63 & 0.55 & 1.03 \\
\hline $8747.34 \ldots \ldots \ldots$ & 12.96 & 0.63 & -0.64 & 0.55 & 1.04 \\
\hline $8802.25 .$. & 12.96 & 0.60 & -0.65 & 0.56 & 1.03 \\
\hline $8803.27 \ldots \ldots \ldots$ & 12.92 & 0.61 & -0.66 & 0.54 & 1.00 \\
\hline $8823.22 .$. & 12.91 & 0.62 & -0.65 & 0.51 & 1.02 \\
\hline
\end{tabular}

TABLE 3

Photolectric Photometry from OAFA (33" aperture)

\begin{tabular}{cccc}
\hline \hline JD $-2,440,000$ & $V$ & $B-V$ & $U-B$ \\
\hline $8638.69 \ldots \ldots \ldots$ & 12.64 & $\ldots$ & $\ldots$ \\
$8653.73 \ldots \ldots \ldots$ & 12.67 & $\ldots$ & $\ldots$ \\
$8653.75 \ldots \ldots \ldots$ & 12.68 & $\ldots$ & $\ldots$ \\
$8661.75 \ldots \ldots \ldots$ & 12.65 & $\ldots$. & $\ldots$ \\
$8663.69 \ldots \ldots \ldots$ & 12.67 & 0.71 & $\ldots$ \\
$8665.76 \ldots \ldots \ldots$ & 12.69 & 0.72 & -0.70 \\
$8665.77 \ldots \ldots \ldots$. & 12.68 & 0.70 & -0.60 \\
$8690.65 \ldots \ldots \ldots$ & 12.72 & 0.71 & -0.50 \\
$8716.66 \ldots \ldots \ldots$. & 12.68 & 0.68 & -0.54 \\
$8743.54 \ldots \ldots \ldots$ & 12.70 & 0.69 & -0.48 \\
$8809.54 \ldots \ldots \ldots$ & 12.61 & 0.66 & -0.61 \\
$8833.52 \ldots \ldots \ldots$ & 12.60 & 0.63 & -0.56 \\
\hline
\end{tabular}

TABLE 4

CCD PHOTOMETRY FROM VBO (18" aperture)

\begin{tabular}{lccc}
\hline \hline JD $-2,440,000$ & $V$ & $B-V$ & $V-R$ \\
\hline $8657.41 \ldots \ldots \ldots$ & 13.00 & 0.51 & 0.63 \\
$8657.44 \ldots \ldots \ldots$ & 12.98 & 0.51 & 0.63 \\
$8688.31 \ldots \ldots \ldots$ & 13.13 & 0.63 & 0.68 \\
$8689.31 \ldots \ldots \ldots$ & 13.13 & 0.61 & 0.67 \\
$8715.24 \ldots \ldots \ldots$ & 13.09 & 0.49 & 0.65 \\
\hline
\end{tabular}

TABLE 5

NEAR-IR OBSERVATIONS FROM SAAO (12" aperture)

\begin{tabular}{|c|c|c|c|c|}
\hline JD $-2,440,000$ & $J$ & $H$ & $K$ & $L$ \\
\hline 8576. & 11.33 & 10.43 & 9.71 & $8.32 \pm 0.06$ \\
\hline $8593 \ldots$ & 11.41 & 10.52 & 9.76 & $8.37 \pm 0.11$ \\
\hline $8629 .$. & 11.33 & 10.44 & 9.70 & $8.40 \pm 0.05$ \\
\hline $8630 .$. & 11.37 & 10.44 & 9.71 & $8.35 \pm 0.06$ \\
\hline $8632 .$. & 11.33 & 10.44 & 9.70 & $8.32 \pm 0.06$ \\
\hline $8633 .$. & 11.35 & 10.43 & 9.70 & $8.34 \pm 0.06$ \\
\hline $8634 \ldots$ & 11.34 & 10.45 & 9.71 & $8.37 \pm 0.05$ \\
\hline 8671. & 11.35 & 10.46 & 9.69 & $8.34 \pm 0.06$ \\
\hline $8674 \ldots$ & 11.32 & 10.42 & 9.67 & $8.29 \pm 0.06$ \\
\hline $8675 \ldots$ & 11.32 & 10.41 & 9.65 & $8.23 \pm 0.06$ \\
\hline 8700 . & 11.40 & 10.45 & 9.70 & $8.39 \pm 0.06$ \\
\hline 8727. & 11.33 & 10.43 & 9.69 & $8.34 \pm 0.05$ \\
\hline $8731 \ldots$ & 11.34 & 10.45 & 9.72 & $8.31 \pm 0.06$ \\
\hline 8733. & 11.35 & 10.43 & 9.69 & $8.38 \pm 0.06$ \\
\hline $8734 .$. & 11.33 & 10.45 & 9.70 & $8.39 \pm 0.06$ \\
\hline $8735 \ldots$ & 11.24 & 10.44 & 9.72 & $8.30 \pm 0.06$ \\
\hline $8768 \ldots \ldots \ldots \ldots$ & 11.28 & 10.47 & 9.72 & $8.36 \pm 0.05$ \\
\hline $8783 .$. & 11.17 & 10.39 & 9.65 & $8.31 \pm 0.06$ \\
\hline $8786 \ldots \ldots$ & 11.09 & 10.27 & 9.62 & $8.32 \pm 0.06$ \\
\hline $8789 \ldots \ldots \ldots \ldots$ & 11.16 & 10.34 & 9.60 & $8.32 \pm 0.08$ \\
\hline $8790 .$. & 11.07 & 10.27 & 9.58 & $8.24 \pm 0.06$ \\
\hline 8792. & 11.16 & 10.35 & 9.63 & $8.27 \pm 0.06$ \\
\hline 8804. & 11.13 & 10.29 & 9.59 & $8.22 \pm 0.05$ \\
\hline 8834. & 11.20 & 10.35 & 9.60 & $8.26 \pm 0.05$ \\
\hline
\end{tabular}


The uncertainty of the $J, H$, and $K$ magnitudes is 0.03 at all epochs. The uncertainties of the $L$ magnitudes are listed in the table.

\section{ABSOLUTE CALIBRATION OF THE SPECTRA}

In order to correct for slit losses, the spectra which include $\mathrm{H} \beta$ were internally calibrated relative to each other with the method described by van Groningen \& Wanders (1992). The method finds the optimum scaling factor, wavelength shift, and convolution factor of one spectrum with respect to another one taken as reference, by slowly varying these parameters until the residuals of one or more constant narrow lines in the difference between the two spectra are minimized. One of the highest quality spectra (Fig. 1), obtained at ESO on 1991 December 18 (JD 2,448,609), was used as reference, and the residuals of the strong lines [O III] $\lambda \lambda 4959,5007$ were minimized. More than $90 \%$ of the multiplicative scaling factors yielded by the scaling program are between 0.8 and 1.5. The accuracy of the internal calibration is $\sim 2 \%$ for most of the spectra, judging from the [O III] residuals obtained when slightly varying the scaling factor. Although the ESO spectra were obtained through a considerably narrower slit, differential light losses caused by the slightly extended [O III] emission (Winge et al. 1992) were negligible: a comparison between subsets of ESO and CTIO line fluxes obtained with separations of 2 days at most showed that any systematic offset between the two data sets is less than $0.5 \%$.

The accuracy of the internal calibration method for spectra of very different resolutions was tested by convolving some of the highest resolution spectra with a Gaussian of appropriate width, in order to simulate the lowest resolution used, and running the internal calibration program again: the resulting scaling factors differed from those obtained for the nonconvolved spectra by $3 \%$ at most, always in excess. This effect is partly compensated, when measuring the line flux (see $\S 5$ ), by the fact that the lower resolution causes a small fraction of the flux to be lost at the edges of the integration interval: the resulting $\mathrm{H} \beta$ flux, therefore, does not differ by more than $2 \%$.

In order to obtain the absolute calibration of the spectra, the flux of [O III] $\lambda 5007$ was measured in the five CTIO spectra in which the [O III] lines were strongest before the internal calibration: these spectra are of the best quality and presumably suffered the least from slit losses or bad observing conditions. The CTIO data set was chosen to obtain the absolute calibration because of its homogeneity and large aperture. The [O III] $\lambda 5007$ measurements were obtained by fitting a straight pseudocontinuum under the line and integrating the flux above it. The fluxes are listed in Table 6, with their $1 \sigma$ uncertainties. The last line of the table gives the mean value and standard deviation of the listed values. We henceforth assume that the flux of [O III] $\lambda 5007$ is $8.44 \times 10^{-13} \mathrm{erg} \mathrm{s}^{-1} \mathrm{~cm}^{-2}$. The [O III] $\lambda 5007$ flux measured in the ESO spectrum used as reference for the internal calibration (JD $2,448,609$ ) is $8.85 \times 10^{-13} \mathrm{erg} \mathrm{s}^{-1}$ $\mathrm{cm}^{-2}$ : therefore, all internally calibrated spectra were multiplied by a factor of 0.95 in order to obtain the right absolute calibration.

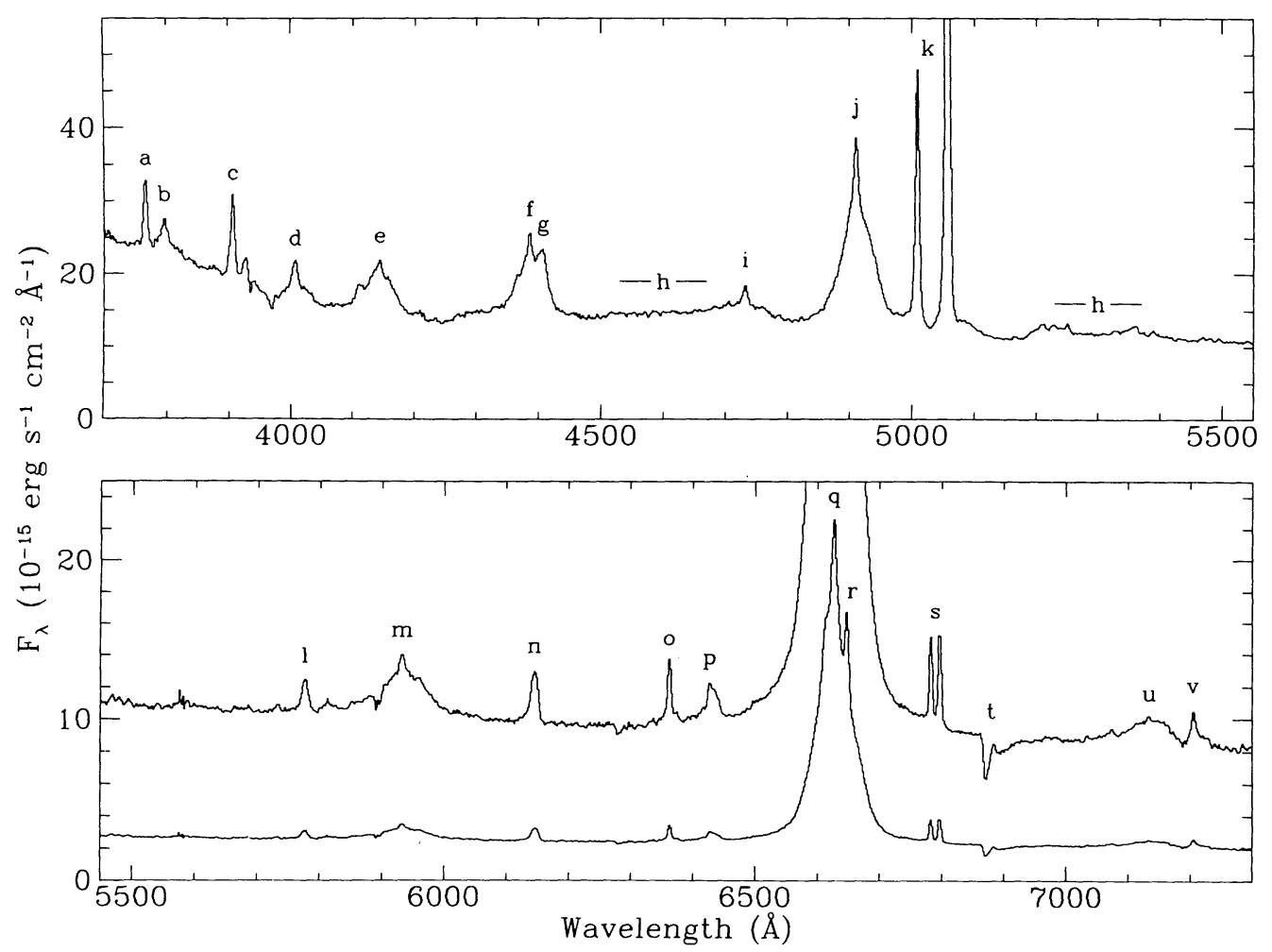

FIG. 1.- Spectrum of NGC 3783 obtained at ESO on 1991 December 18/19 (JD 2,448,609). The wavelength range was covered entirely by a single exposure, and

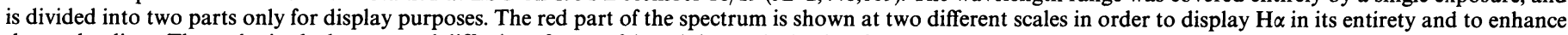

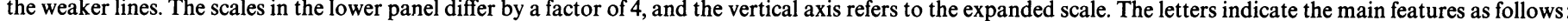

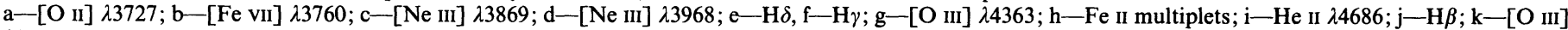

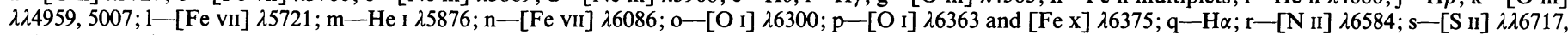
$6731 ; \mathrm{t}-\mathrm{O}_{2} B$ band (telluric); u- $-\mathrm{He}_{\mathrm{I}} \lambda 7065 ; \mathrm{v}-[\mathrm{Ar} \mathrm{III}] \lambda 7136$. 
TABLE 6

Absolute Flux of [O III] $\lambda 5007$

\begin{tabular}{cc}
\hline \hline JD $-2,440,000$ & $\begin{array}{c}\text { Flux } \\
\left(10^{-13} \mathrm{erg} \mathrm{s}^{-1} \mathrm{~cm}^{-2}\right)\end{array}$ \\
\hline $8607 \ldots \ldots \ldots \ldots \ldots$ & $8.44 \pm 0.17$ \\
$8651 \ldots \ldots \ldots \ldots \ldots$. & $8.36 \pm 0.17$ \\
$8678 \ldots \ldots \ldots \ldots \ldots$. & $8.52 \pm 0.17$ \\
$8716 \ldots \ldots \ldots \ldots \ldots$. & $8.39 \pm 0.17$ \\
$8724 \ldots \ldots \ldots \ldots \ldots$ & $8.46 \pm 0.17$ \\
Mean $\ldots \ldots \ldots \ldots$. & $8.435 \pm 0.059$ \\
\hline
\end{tabular}

The value thus obtained for the [O III] $\lambda 5007$ flux is about $15 \%$ less than the flux measured by Osmer, Smith, \& Weedman (1974), and we will therefore assume that the systematic uncertainty of our absolute fluxes is of this order.

\section{THE CONTINUUM LIGHT CURVES}

\subsection{Optical Continuum}

The light curve of the optical continuum was obtained by combining spectral with photometric data. The continuum measurements must not be significantly contaminated by varying emission lines, which could introduce spurious delay in the continuum variations: care was taken, therefore, to use a part of the spectrum and a photometric band which are influenced as little as possible by the broad lines. For the spectra, an additional constraint is given by the necessity of obtaining the measurements in a region close to the [O III] lines, used as internal calibrators, so that inaccuracies in the response calibration do not introduce further uncertainties in the light curve.

The continuum flux was derived from the spectra by averaging the flux density in an interval of $20 \AA$ centered at $5150 \AA$, i.e., on the dip between the lines Fe II $\lambda \lambda 5018$ and 5169 , which is considered to be the region close to $\mathrm{H} \beta$ least contaminated by emission lines (Fig. 2). Although the two Fe II lines may have slightly overlapping wings in this interval, their variations have a negligible influence on the continuum light curve.

The continuum measurements include a contribution from the underlying galaxy, which has a different spatial distribution than the nucleus: the galaxy has an apparent diameter of $\sim 2$, while the broad-line region (BLR) and the region which emits the nonstellar continuum are unresolved. Therefore, only the CTIO and OSU spectra were used, because of their similar large apertures: a wide slit, in fact, minimizes the differential light losses caused by the different spatial profiles of the nucleus and the underlying galaxy, and therefore ensures that the contribution of the stellar component to the continuum fluxes depends only weakly on the seeing value. Because the seeing was always lower than $2^{\prime \prime}$ during the CTIO observations, the stellar component can be considered constant within the uncertainties.

The greatest source of uncertainty in the flux measurements is the internal calibration error, which in all spectra of good quality is considerably larger than the error introduced by the photon noise. Accordingly, most uncertainties were set at $2 \%$. For some fluxes, obtained from spectra with lower signal-tonoise ratio, the error bars were set at $4 \%$.

These points were then combined with the $V$ fluxes, taken to represent measurements of the optical continuum: this band was chosen not only because its central wavelength is the closest to $5150 \AA$ but also because it samples a section of the optical spectrum which is almost free from strong variable emission lines. The strongest broad lines are He I $\lambda 5876$ and the Fe II blend centered at $5250 \AA$ (rest wavelength). The equivalent widths of these features in the CTIO spectra are less than

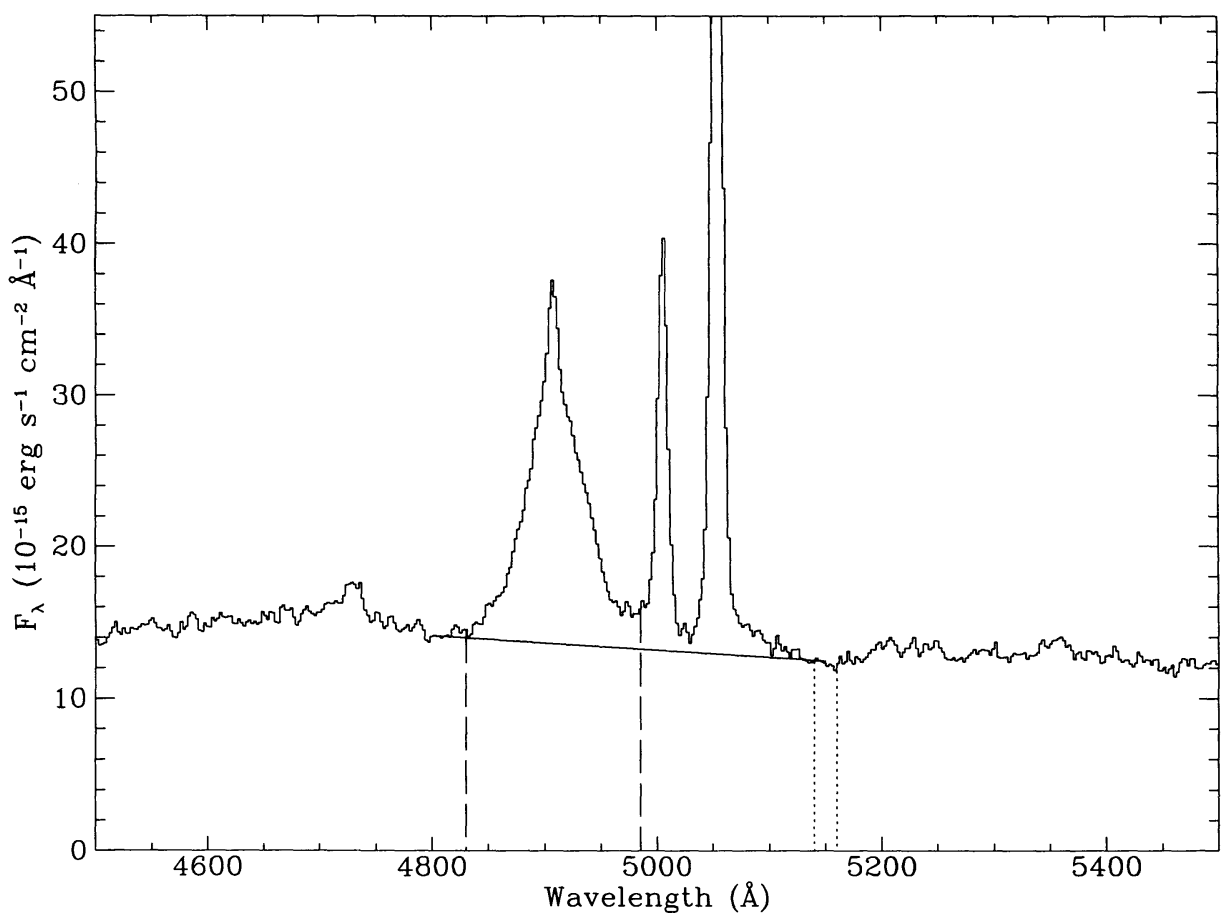

FIG. 2.-H $\beta$ region of the spectrum obtained at CTIO on 1992 February 11/12 (JD 2,448,664). The straight lines illustrate the method used to measure the fluxes of $\mathrm{H} \beta$ and of the continuum: the continuous line indicates the straight-line fit which approximates the continuum under $\mathrm{H} \beta$; the dashed lines indicate the limits of the line flux integration above the continuum fit; and the dotted lines indicate the limits of the interval used to average the continuum flux. 
$40 \AA$ and less than $25 \AA$, respectively: we can therefore assume that the variations of these lines, and their possible delays with respect to those of the continuum, have a negligible effect on the $V$ light curve. The main narrow lines present in the $V$ band are [Fe VII] $\lambda 5721$, which is weak, and the [O III] lines: the latter introduce at most only a slight constant offset in the $V$ curve.

The $V$ magnitudes were converted to flux densities using the relation

$$
F_{\lambda}=10^{(-0.4 V-8.47)}
$$

(Hayes \& Latham 1975), with $F_{\lambda}$ in $\mathrm{erg} \mathrm{s}^{-1} \mathrm{~cm}^{-2} \AA^{-1}$. Although the spectrum of NGC 3783 does not have prominent emission features in the $V$ band, this conversion may nevertheless be affected by a systematic error because of the differences between the spectrum of an AGN and that of the star (Vega) used to define relation (1). Furthermore, as explained in $\S 3$, the spectroscopic continuum fluxes have a systematic uncertainty of $15 \%$. For both these reasons the continuum flux densities obtained from different data sets were compared, before being combined in a single optical continuum curve, as follows.

If the conversion of the photometric $V$ data to flux densities is correct, and if the absolute calibration of the spectroscopic fluxes is accurate, pairs of quasi-simultaneous fluxes from one of the photometric data sets and from the spectroscopic data set should align along a line of slope 1 , and the photometric fluxes should have a positive offset caused by the underlying galaxy's higher contribution through the larger aperture. For each photometric data set, pairs formed by a photometric and a spectroscopic flux separated by at most 1 day are plotted in Figure 3. In each panel the continuous line is the line of slope 1 which best fits the data. For the first two subsets (SAAO versus spectroscopy and OAFA versus spectroscopy), two leastsquares linear regression fits were calculated: ordinates against abscissae and vice versa. As shown in Figure 3, the slope of the bisector of the fits is close to unity for both subsets. Using the least-squares fit bisector to fine-tune the conversion would change the photometric fluxes by $1 \sigma$ at most. The fits were not calculated for the VBO versus spectroscopy subset, as it consists of only three points. Figure 3 shows that the relation between these points is also well represented by a line of slope 1 .

This comparison of quasi-simultaneous pairs of points also allowed us to correct the photometric fluxes, in order to account for the different apertures used. The underlying galaxy contributes in different amounts to each data set. In particular, the photometric measurements, obtained through apertures with diameters of $18^{\prime \prime}, 20^{\prime \prime}$, and $33^{\prime \prime}$, are much higher than the spectroscopic fluxes, obtained through a $5^{\prime \prime} \times 10^{\prime \prime}$ rectangular aperture. The comparison of quasi-simultaneous pairs of points yielded mean offsets of $(8.62 \pm 0.74) \times 10^{-15} \mathrm{erg} \mathrm{s}^{-1}$ $\mathrm{cm}^{-2} \AA^{-1}$ for the VBO fluxes, $(9.71 \pm 0.66) \times 10^{-15} \mathrm{erg} \mathrm{s}^{-1}$ $\mathrm{cm}^{-2} \AA^{-1}$ for the SAAO fluxes, and $(16.64 \pm 0.51) \times 10^{-15}$ $\mathrm{erg} \mathrm{s}^{-1} \mathrm{~cm}^{-2} \AA^{-1}$ for the OAFA fluxes. These quantities were subtracted from the photometric data, thus simulating photometry through a $5^{\prime \prime} \times 10^{\prime \prime}$ rectangular aperture. The correction also automatically eliminates the constant offset which may be caused by the presence of the [O III] lines in the $V$ band (see above). All fluxes are obviously still contaminated by stellar light from the underlying galaxy: given the large aperture, this component can be considered independent of seeing effects and therefore constant. As discussed in detail by Alloin et al. (1994), examination of the two-dimensional spectra made it possible

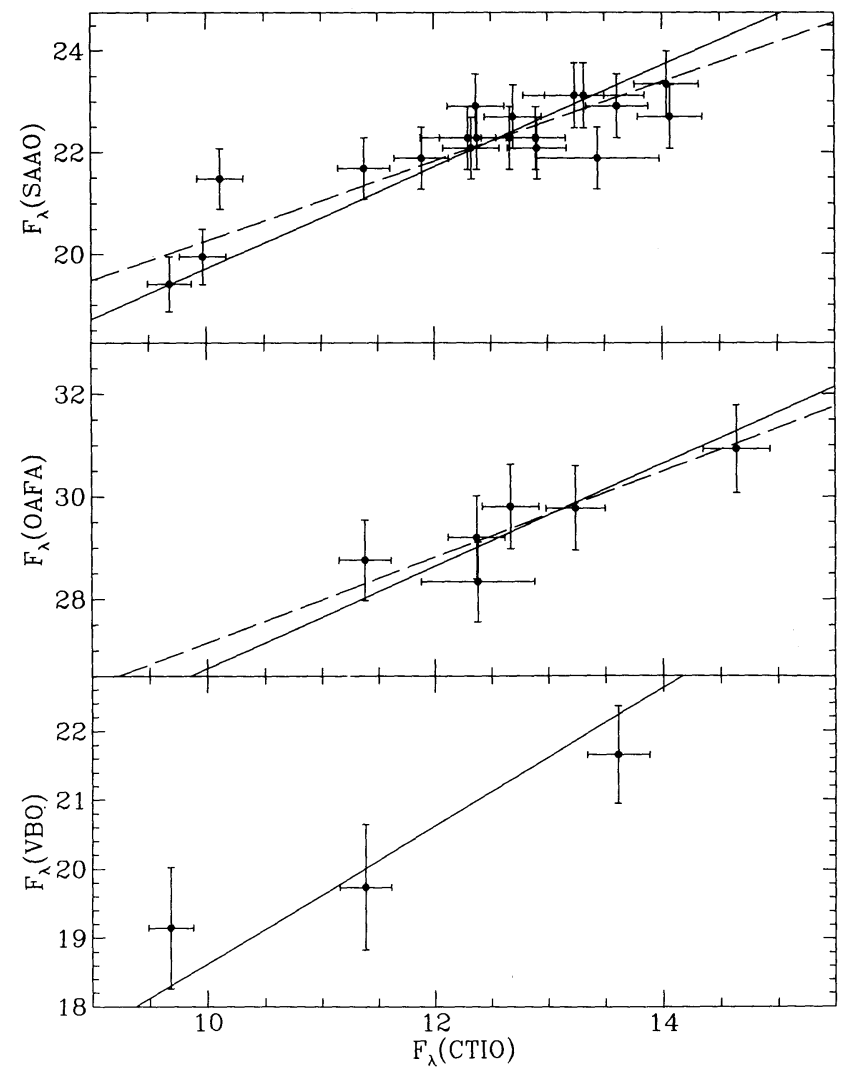

FIG. 3-Comparison between quasi-simultaneous subsets of continuum fluxes obtained from the spectroscopic (CTIO) and photometric (SAAO, OAFA, and VBO) data sets. Units are $10^{-15} \mathrm{erg} \mathrm{s}^{-1} \AA^{-1}$. For each plotted point the corresponding observations were separated in time by 1 day at most. The photometric flux densities were obtained from the $V$ data using equation (1). The solid lines have slope 1 and intercept the $y$-axis at the mean difference between ordinates and abscissae. This offset between different data sets is caused by the larger apertures used for the photometry, and therefore to a larger flux from the underlying galaxy. The dashed lines represent the bisectors of the least-squares linear regression fits calculated for ordinates against abscissae and vice versa.

to estimate that the stellar contribution is about $30 \%$ in the CTIO continuum measurements.

The continuum light curve obtained by combining the spectroscopic and photometric data is listed in Table 7 and plotted in Figure 4. Fluxes obtained at the same date were averaged and their uncertainties averaged quadratically. The median separation between consecutive points of the curve is 2.0 days. The variations of the optical continuum strongly resemble those of the UV continuum (Paper V).

\subsection{Near-Infrared Continuum}

Figure 5 shows the IR light curves obtained at SAAO: the magnitudes were converted to flux densities using the relations

$$
\begin{gathered}
F_{\lambda}(J)=10^{(-0.4 J-9.54)}, \quad F_{\lambda}(K)=10^{(-0.4 K-9.94),} \\
F_{\lambda}(H)=10^{(-0.4 H-10.42)}, \quad F_{\lambda}(L)=10^{(-0.4 L-11.16),}
\end{gathered}
$$

with $F_{\lambda}$ in $\mathrm{erg} \mathrm{s}^{-1} \mathrm{~cm}^{-2} \AA^{-1}$. The IR flux showed little evidence for variation during the monitoring period and was generally lower than the long-term average in the $K(2.2 \mu \mathrm{m})$ and $L$ $(3.5 \mu \mathrm{m})$ bands. The only significant change was an increase of $\sim 20 \%$ at $J, H$, and $K$ and $\sim 10 \%$ at $L$ between JD 2,448,768 and JD 2,448,790. The wavelength trend of this variation was 
TABLE 7

Optical ContinuUm light Curve

\begin{tabular}{|c|c|c|c|c|c|}
\hline JD $-2,440,000$ & $\begin{array}{c}F_{\lambda}(\text { opt }) \\
\left(10^{-15} \mathrm{erg} \mathrm{s}^{-1} \mathrm{~cm}^{-2} \AA^{-1}\right)\end{array}$ & Source & JD $-2,440,000$ & $\begin{array}{c}F_{\lambda}(\mathrm{opt}) \\
\left(10^{-15} \mathrm{erg} \mathrm{s}^{-1} \mathrm{~cm}^{-2} \AA^{-1}\right)\end{array}$ & Source \\
\hline 8607.83 . & $13.02 \pm 0.26$ & A & 8690.65 . & $11.06 \pm 0.76$ & $F$ \\
\hline $8610.56 \ldots \ldots \ldots$ & $14.19 \pm 0.63$ & G & $8700.45 \ldots \ldots \ldots$ & $10.79 \pm 0.57$ & G \\
\hline $8622.55 \ldots$ & $12.57 \pm 0.62$ & G & $8704.50 .$. & $10.00 \pm 0.20$ & $\mathrm{~A}, \mathbf{G}$ \\
\hline $8623.83 \ldots$ & $12.90 \pm 0.26$ & A & 8712.59. & $11.78 \pm 0.24$ & $\mathrm{~A}$ \\
\hline $8627.83 .$. & $12.35 \pm 0.25$ & A & $8714.41 .$. & $11.18 \pm 0.58$ & G \\
\hline $8630.57 \ldots$ & $12.57 \pm 0.62$ & G & $8715.24 \ldots$ & $11.11 \pm 0.91$ & $\mathbf{H}$ \\
\hline 8631.84 .. & $12.30 \pm 0.25$ & A & 8716.60 .. & $11.44 \pm 0.23$ & $\mathrm{~A}, \mathrm{~F}$ \\
\hline $8634.55 .$. & $12.16 \pm 0.61$ & G & 8717.50 . & $11.96 \pm 0.60$ & G \\
\hline $8635.83 \ldots$ & $11.89 \pm 0.24$ & $\mathbf{A}$ & $8720.59 .$. & $13.04 \pm 0.26$ & A \\
\hline $8638.63 \ldots$ & $12.79 \pm 0.50$ & $F, G$ & $8724.56 .$. & $12.81 \pm 0.26$ & A \\
\hline $8639.84 .$. & $12.67 \pm 0.25$ & A & $8728.55 .$. & $12.91 \pm 0.26$ & A \\
\hline $8643.72 .$. & $14.07 \pm 0.28$ & $\mathbf{A}$ & 8729.38.. & $12.36 \pm 0.61$ & G \\
\hline $8644.56 \ldots \ldots \ldots$ & $12.98 \pm 0.63$ & G & $8732.56 .$. & $12.33 \pm 0.25$ & A \\
\hline $8647.76 \ldots \ldots \ldots$ & $13.91 \pm 0.28$ & A & $8733.36 \ldots$ & $12.36 \pm 0.61$ & G \\
\hline $8651.67 \ldots$ & $12.75 \pm 0.26$ & $\mathrm{~A}, \mathrm{G}$ & 8736.55 . & $11.64 \pm 0.23$ & $\mathbf{A}$ \\
\hline $8653.74 \ldots \ldots \ldots$ & $12.30 \pm 0.57$ & $\mathbf{F}$ & $8742.67 .$. & $12.21 \pm 0.24$ & B \\
\hline $8655.58 \ldots \ldots \ldots$ & $13.19 \pm 0.63$ & G & $8743.40 .$. & $12.24 \pm 0.48$ & F, G \\
\hline $8656.77 .$. & $13.61 \pm 0.27$ & A & $8744.61 \ldots$ & $12.38 \pm 0.50$ & A \\
\hline $8657.42 \ldots$ & $13.04 \pm 0.71$ & $\mathbf{H}$ & 8747.34.. & $12.57 \pm 0.62$ & G \\
\hline $8659.54 \ldots$ & $13.40 \pm 0.64$ & G & $8752.57 .$. & $12.68 \pm 0.25$ & A \\
\hline $8660.77 \ldots \ldots \ldots$ & $13.24 \pm 0.26$ & A & $8763.58 \ldots$ & $12.89 \pm 0.52$ & $\mathbf{A}$ \\
\hline $8661.75 \ldots$ & $13.15 \pm 0.82$ & $\mathbf{F}$ & $8764.56 \ldots$ & $12.10 \pm 0.24$ & A \\
\hline $8663.53 \ldots$ & $12.96 \pm 0.50$ & $\mathbf{F}, \mathbf{G}$ & $8772.63 .$. & $13.47 \pm 0.27$ & A \\
\hline $8664.77 \ldots \ldots \ldots$ & $12.37 \pm 0.25$ & A & $8776.56 \ldots$ & $11.71 \pm 0.23$ & A \\
\hline $8665.77 \ldots$ & $12.03 \pm 0.56$ & $\mathbf{F}$ & $8794.57 .$. & $12.76 \pm 0.26$ & A \\
\hline $8668.81 \ldots$ & $11.31 \pm 0.23$ & A & $8802.25 \ldots$ & $12.57 \pm 0.62$ & G \\
\hline $8671.52 \ldots \ldots \ldots$ & $12.16 \pm 0.61$ & G & $8803.27 \ldots$ & $13.40 \pm 0.64$ & G \\
\hline $8672.72 \ldots$ & $13.44 \pm 0.54$ & A & $8804.47 .$. & $13.32 \pm 0.53$ & A \\
\hline $8676.71 \ldots$ & $11.97 \pm 0.24$ & A & $8809.54 \ldots$ & $14.18 \pm 0.85$ & $\mathrm{~F}$ \\
\hline $8677.80 \ldots$ & $12.28 \pm 0.25$ & A & $8822.48 \ldots$ & $14.68 \pm 0.29$ & A \\
\hline $8678.68 \ldots$ & $12.09 \pm 0.24$ & A & $8823.22 .$. & $13.62 \pm 0.65$ & G \\
\hline $8684.52 \ldots$ & $11.76 \pm 0.59$ & $\mathbf{G}$ & 8824.47 .. & $13.41 \pm 0.27$ & $\bar{A}$ \\
\hline $8685.80 \ldots$ & $10.12 \pm 0.20$ & $\mathbf{A}^{\prime}$ & $8826.47 .$. & $14.36 \pm 0.29$ & A \\
\hline $8687.74 \ldots$ & $9.68 \pm 0.19$ & $\mathrm{~A}^{\prime \prime}$ & $8830.48 \ldots \ldots \ldots$ & $13.32 \pm 0.27$ & A \\
\hline $8688.60 \ldots$ & $9.92 \pm 0.46$ & $\mathbf{G}, \mathbf{H}$ & $8832.46 .$. & $14.64 \pm 0.29$ & $\mathbf{A}$ \\
\hline $8689.31 \ldots \ldots \ldots$ & $10.45 \pm 0.88$ & $\mathbf{H}$ & $8833.52 \ldots$ & $14.29 \pm 0.85$ & $\mathbf{F}$ \\
\hline
\end{tabular}

NotE-Codes for data origin: A-CTIO spectroscopy; B-OSU spectroscopy; F-OAFA photoelectric photometry; G-SAAO photoelectric photometry; H-VBO CCD photometry.

different from that described by Glass (1992), who found that the amplitude of variation (in magnitudes and flux units) increases with wavelength in the JHKL bands.

There is no obvious similarity between the near-IR light curves and the UV and optical ones. However, one should consider that the former are sampled more sparsely, and that we expect them to be smoother than the UV and optical curves because the region which emits the near-IR flux is likely to be much larger than the BLR (Clavel, Wamsteker, \& Glass 1989; Glass 1992). Given the limited amplitude and duration of the UV and optical variations, we cannot exclude that corresponding features have simply been smoothed out of the near-IR light curves.

There is no doubt as to the reality of the increase of flux around JD $2,448,780$, but the spectral shape of the event is distinctly peculiar. The new $H$ and $K$ flux observations have been plotted on Figure $2 b$ of Glass (1992), and three of them (JD 2,448,786, JD 2,448,790, and JD 2,448,804, which are also the brightest in $J$ and $H$ ) fall significantly away from the general trend, which is that $H$ and $K$ are always linearly related. The reality of this interesting behavior must at present be considered provisional. A large number of observers were involved in the work, and small systematic differences between them cannot be entirely excluded.

\section{THE LIGHT CURVE OF $\mathrm{H} \beta$}

The flux of $\mathrm{H} \beta$ was measured as for NGC 5548 in Paper II, by fitting a straight-line continuum in the intervals $4800-4820$ $\AA$ and $5130-5150 \AA$, and integrating the flux above the line between 4830 and $4985 \AA$ (Fig. 2). The constant narrow component of $\mathrm{H} \beta$ is included in the measurements: its contribution to the total line flux is $\sim 7 \times 10^{-14} \mathrm{erg} \mathrm{s}^{-1} \mathrm{~cm}^{-2}(6 \%-8 \%$ of the broad-line flux, depending on the latter's strength).

Winge et al. (1992) have shown that the $\mathrm{H} \alpha$ emission extends to apparent distances of $\sim 10^{\prime \prime}$ from the nucleus: there is therefore a possibility that the $\mathrm{H} \beta$ emission is also extended, and that seeing effects and different slit widths may influence the $\mathrm{H} \beta$ fluxes. The internal calibration is not affected, because only the $[\mathrm{O} \mathrm{III}]$ lines were used for its determination. The broad-line component (which forms the bulk of $\mathrm{H} \beta$ ) is emitted by an unresolved region, which means that the broad-line fluxes are not affected either. Any extended $\mathrm{H} \beta$ emission would only influence the narrow-line component, causing its flux to increase with increasing aperture and seeing. A strong effect is unlikely, since the narrow component is only a small fraction of the measured $\mathrm{H} \beta$ flux. The test described in $\S 3$ (the comparison of subsets of quasi-simultaneous $\mathrm{H} \beta$ fluxes from CTIO and ESO spectra) yields a negligible offset between the two subsets. 


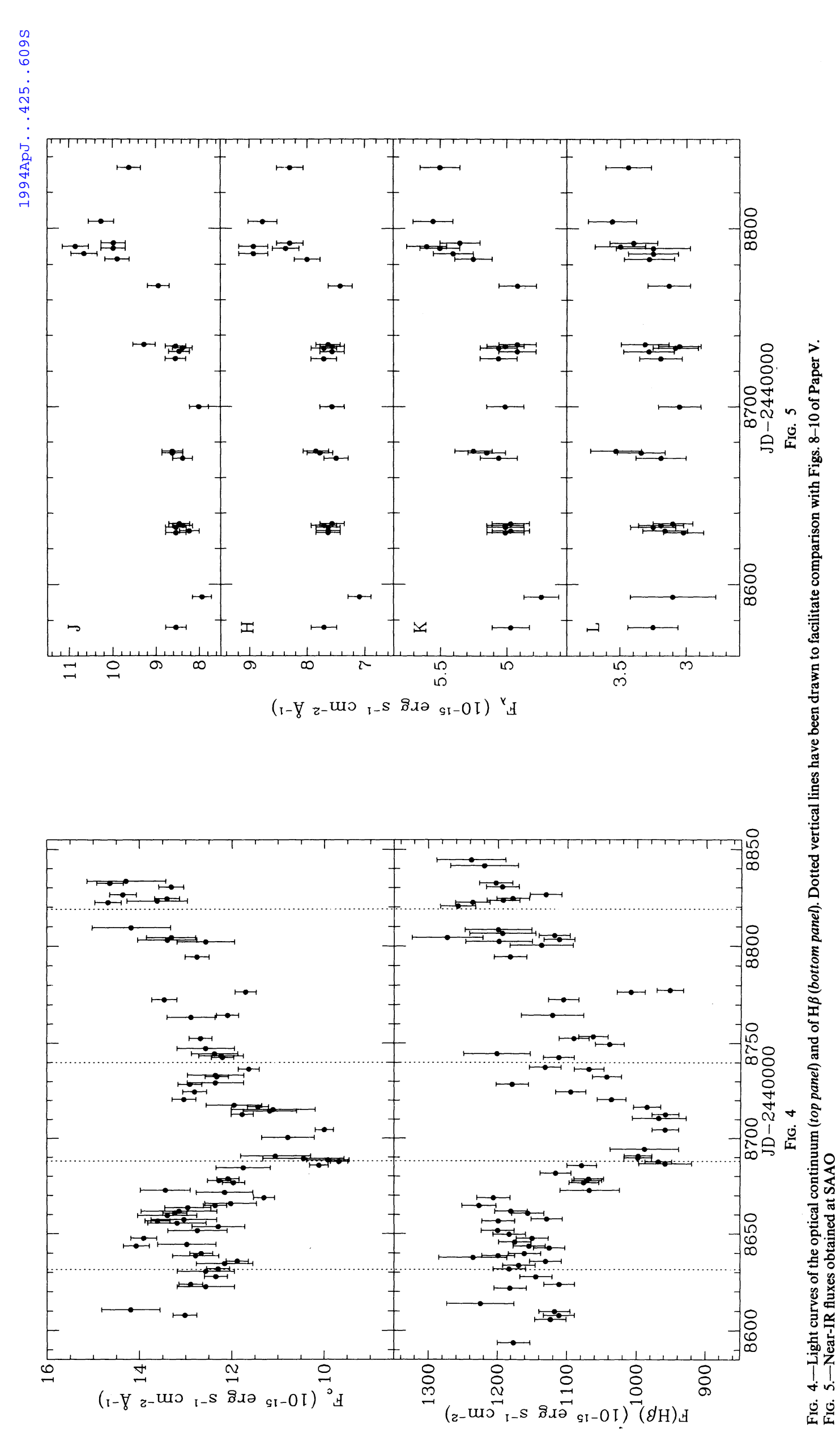

(C) American Astronomical Society - Provided by the NASA Astrophysics Data System 
TABLE 8

H $\beta$ Light CURVE

\begin{tabular}{|c|c|c|c|c|c|}
\hline $\mathrm{JD}-2,440,000$ & $\begin{array}{c}F(\mathbf{H} \beta) \\
\left(10^{-15} \mathrm{erg} \mathrm{s}^{-1} \mathrm{~cm}^{-2}\right)\end{array}$ & Source & JD $-2,440,000$ & $\begin{array}{c}F(\mathrm{H} \beta) \\
\left(10^{-15} \mathrm{erg} \mathrm{s}^{-1} \mathrm{~cm}^{-2}\right)\end{array}$ & Source \\
\hline 8593.83. & $1176 \pm 24$ & D & 8704.58 . & $958+19$ & $\mathbf{A}$ \\
\hline $8605.83 \ldots$ & $1123 \pm 22$ & D & 8710.67 & $967 \pm 39$ & D \\
\hline $8607.83 \ldots$ & $1111 \pm 22$ & $\mathbf{A}$ & 8712.59 . & $958 \pm 19$ & A \\
\hline $8609.81 \ldots$ & $1118 \pm 22$ & D & $8716.60 \ldots$ & $985 \pm 20$ & A \\
\hline $8613.84 \ldots \ldots \ldots$ & $1225 \pm 49$ & $\mathbf{D}^{\prime}$ & $8720.59 \ldots \ldots \ldots$ & $1036 \pm 21$ & A \\
\hline $8621.83 \ldots \ldots \ldots$ & $1182 \pm 24$ & $\mathrm{D}$ & $8724.56 \ldots \ldots \ldots$ & $1095 \pm 22$ & A \\
\hline $8623.83 \ldots$ & $1111 \pm 22$ & A & 8728.55 & $1179 \pm 24$ & A \\
\hline $8627.83 \ldots \ldots \ldots$ & $1144 \pm 23$ & A & $8732.56 \ldots \ldots \ldots$ & $1042 \pm 21$ & A \\
\hline $8631.84 \ldots \ldots \ldots$ & $1183 \pm 24$ & A & $8736.55 \ldots \ldots \ldots$ & $1068 \pm 21$ & A \\
\hline $8633.81 \ldots$ & $1169 \pm 23$ & C, D & $8737.55 \ldots$ & $1131 \pm 23$ & D \\
\hline $8635.83 \ldots$ & $1130 \pm 23$ & A & 8742.67 & $1112 \pm 22$ & B \\
\hline $8637.78 \ldots$ & $1235 \pm 49$ & $\mathrm{C}$ & $8744.61 \ldots \ldots \ldots$ & $1201 \pm 48$ & A \\
\hline $8638.75 \ldots \ldots \ldots$ & $1199 \pm 24$ & $\mathrm{C}$ & $8749.52 \ldots \ldots \ldots$ & $1039 \pm 21$ & D \\
\hline $8639.84 \ldots$ & $1161 \pm 23$ & $\mathrm{~A}$ & $8752.57 \ldots$ & $1090 \pm 22$ & $\mathrm{~A}$ \\
\hline $8642.78 \ldots$ & $1125 \pm 23$ & $\mathrm{C}$ & 8753.54 & $1063 \pm 21$ & D \\
\hline $8643.72 \ldots \ldots \ldots$ & $1154 \pm 23$ & A & $8764.56 \ldots \ldots \ldots$ & $1121 \pm 45$ & A \\
\hline $8645.82 \ldots \ldots \ldots$ & $1174 \pm 23$ & D & $8772.63 \ldots \ldots \ldots$ & $1106 \pm 22$ & A \\
\hline $8647.76 \ldots$ & $1150 \pm 23$ & $\mathbf{A}$ & $8776.56 \ldots$ & $1009 \pm 20$ & A \\
\hline $8649.83 \ldots$ & $1183 \pm 24$ & D & $8777.49 .$. & $952 \pm 19$ & D \\
\hline $8651.76 \ldots \ldots \ldots$ & $1199 \pm 24$ & A & $8794.57 \ldots \ldots \ldots$ & $1182 \pm 24$ & A \\
\hline $8656.77 \ldots \ldots \ldots$ & $1198 \pm 24$ & A & $8800.52 \ldots \ldots \ldots$ & $1137 \pm 45$ & D \\
\hline $8657.86 \ldots \ldots \ldots$ & $1129 \pm 23$ & D & $8802.5 \ldots \ldots \ldots$ & $1198 \pm 48$ & D \\
\hline $8660.77 \ldots \ldots \ldots$ & $1156 \pm 23$ & A & $8803.5 \ldots$ & $1112 \pm 22$ & D \\
\hline $8661.85 \ldots \ldots \ldots$ & $1181 \pm 24$ & D & $8804.47 \ldots \ldots \ldots$ & $1273 \pm 51$ & $\mathrm{~A}$ \\
\hline $8664.77 \ldots \ldots \ldots$ & $1227 \pm 25$ & A & 8805.5 & $1119 \pm 22$ & D \\
\hline $8668.81 \ldots \ldots \ldots$ & $1207 \pm 24$ & A & $8806.57 \ldots$ & $1194 \pm 48$ & A \\
\hline $8672.72 \ldots \ldots \ldots$ & $1067 \pm 43$ & A & $8808.47 \ldots \ldots$ & $1199 \pm 48$ & A \\
\hline $8676.71 \ldots$ & $1075 \pm 22$ & A & $8820.55 \ldots \ldots \ldots$ & $1258 \pm 25$ & D \\
\hline $8677.80 \ldots \ldots \ldots$ & $1071 \pm 21$ & A & $8822.51 \ldots \ldots \ldots$ & $1236 \pm 25$ & A, D \\
\hline $8678.68 \ldots \ldots \ldots$ & $1068 \pm 21$ & A & $8823.50 \ldots \ldots$ & $1193 \pm 24$ & $\mathrm{D}$ \\
\hline $8681.71 \ldots \ldots \ldots$ & $1117 \pm 22$ & D & $8824.50 \ldots \ldots$ & $1178 \pm 24$ & A, D \\
\hline $8685.79 \ldots \ldots \ldots$ & $1078 \pm 22$ & $\mathbf{A}^{\prime}, \mathbf{D}$ & $8826.47 \ldots \ldots$ & $1131 \pm 23$ & A \\
\hline $8686.70 \ldots \ldots \ldots$ & $958 \pm 38$ & $\mathrm{C}$ & $8830.47 \ldots \ldots$ & $1194 \pm 24$ & A \\
\hline $8687.74 \ldots \ldots \ldots$ & $968 \pm 19$ & $\mathrm{~A}^{\prime \prime}$ & $8832.46 \ldots \ldots$ & $1203 \pm 24$ & A \\
\hline $8689.77 \ldots \ldots \ldots$ & $998 \pm 20$ & C, D & $8841.49 \ldots \ldots$ & $1219 \pm 49$ & D \\
\hline $8690.78 \ldots \ldots \ldots$ & $998 \pm 20$ & $\mathrm{C}$ & $8844.50 \ldots \ldots \ldots$ & $1239 \pm 50$ & D \\
\hline $8694.31 \ldots \ldots \ldots$ & $988 \pm 49$ & $\mathrm{E}$ & & & \\
\hline
\end{tabular}

NoTE.-Codes for origin of data as in Table 1.

As the data sets were obtained with different apertures, we conclude that any extended narrow $\mathrm{H} \beta$ emission adds a negligible contribution to spectra obtained through large apertures.

The effect of a low resolution on the line flux measurement was simulated by artificially degrading the spectra with the highest resolution, as described in $\S 3$, and repeating the measurement: the $\mathbf{H} \beta$ flux always decreased, but never by more than $1 \%$. The $1 \sigma$ error bars were estimated as for the continuum fluxes obtained from the spectra (§ 4).

The resulting fluxes and uncertainties are listed in Table 8. Again, fluxes obtained during the same night were averaged. Figure 4 shows the $\mathbf{H} \beta$ light curve, in which the median separation between consecutive points is 2.1 days. As for the optical continuum, the similarity to the $I U E$ light curves (Paper $\mathrm{V})$ is evident.

\section{ANALYSIS OF THE LIGHT CURVES}

Inspection of the spectra already reveals that significant variability took place in the optical band. As an example, Figure 6 illustrates how the continuum and broad lines underwent a strong decrease between JD 2,448,661 and JD $2,448,704$. Table 9 gives a summary of the main variability characteristics of the optical and IR continua and of $\mathrm{H} \beta$, calculated as in Paper V: the table lists the mean value of each curve (each point was weighted with the inverse square of its uncertainty), the reduced $\chi^{2}$ for variability with respect to the mean, and the ratio between the maximum and minimum fluxes, $R_{\max }$. The variability is significant in all light curves except that of the $L$-band flux. The optical continuum varied with a lower amplitude than the UV continua (Paper V), and the multicolor photometry obtained at SAAO, OAFA, and

TABLE 9

VARIABILITY PARAMETERS

\begin{tabular}{cccc}
\hline \hline Feature & Mean Flux $^{\mathrm{a}}$ & \multicolumn{1}{c}{$\chi_{v}^{2 \mathrm{~b}}$} & \multicolumn{1}{c}{$R_{\max }^{\mathrm{c}}$} \\
\hline$F_{\lambda}(\mathrm{opt}) \ldots \ldots \ldots \ldots$ & 12.4 & 12.0 & $1.5 \pm 0.1$ \\
$F_{\lambda}(J) \ldots \ldots \ldots \ldots$ & 8.8 & 10.0 & $1.4 \pm 0.1$ \\
$F_{\lambda}(H) \ldots \ldots \ldots \ldots$ & 7.8 & 4.2 & $1.3 \pm 0.1$ \\
$F_{\lambda}(K) \ldots \ldots \ldots \ldots$ & 5.1 & 2.5 & $1.2 \pm 0.1$ \\
$F_{\lambda}(L) \ldots \ldots \ldots \ldots$ & 3.4 & 0.8 & $1.2 \pm 0.1$ \\
$F(\mathrm{H} \beta) \ldots \ldots \ldots \ldots$ & 1109 & 11.2 & $1.3 \pm 0.1$ \\
\hline
\end{tabular}

${ }^{\text {a }}$ Weighted mean. Units are $10^{-15} \mathrm{erg} \mathrm{s}^{-1} \mathrm{~cm}^{-2} \AA^{-1}$ for continuum light curves and $10^{-15} \mathrm{erg} \mathrm{s}^{-1} \mathrm{~cm}^{-2}$ for $\mathrm{H} \beta$ light curve.

b Reduced $\chi^{2}$ for variability about mean. Degrees of freedom are 71 for optical continuum, 23 for IR continua, and 72 for $\mathbf{H} \beta$.

c Ratio between maximum and minimum values. Uncertainties calculated by propagation of $1 \sigma$ flux errors. 


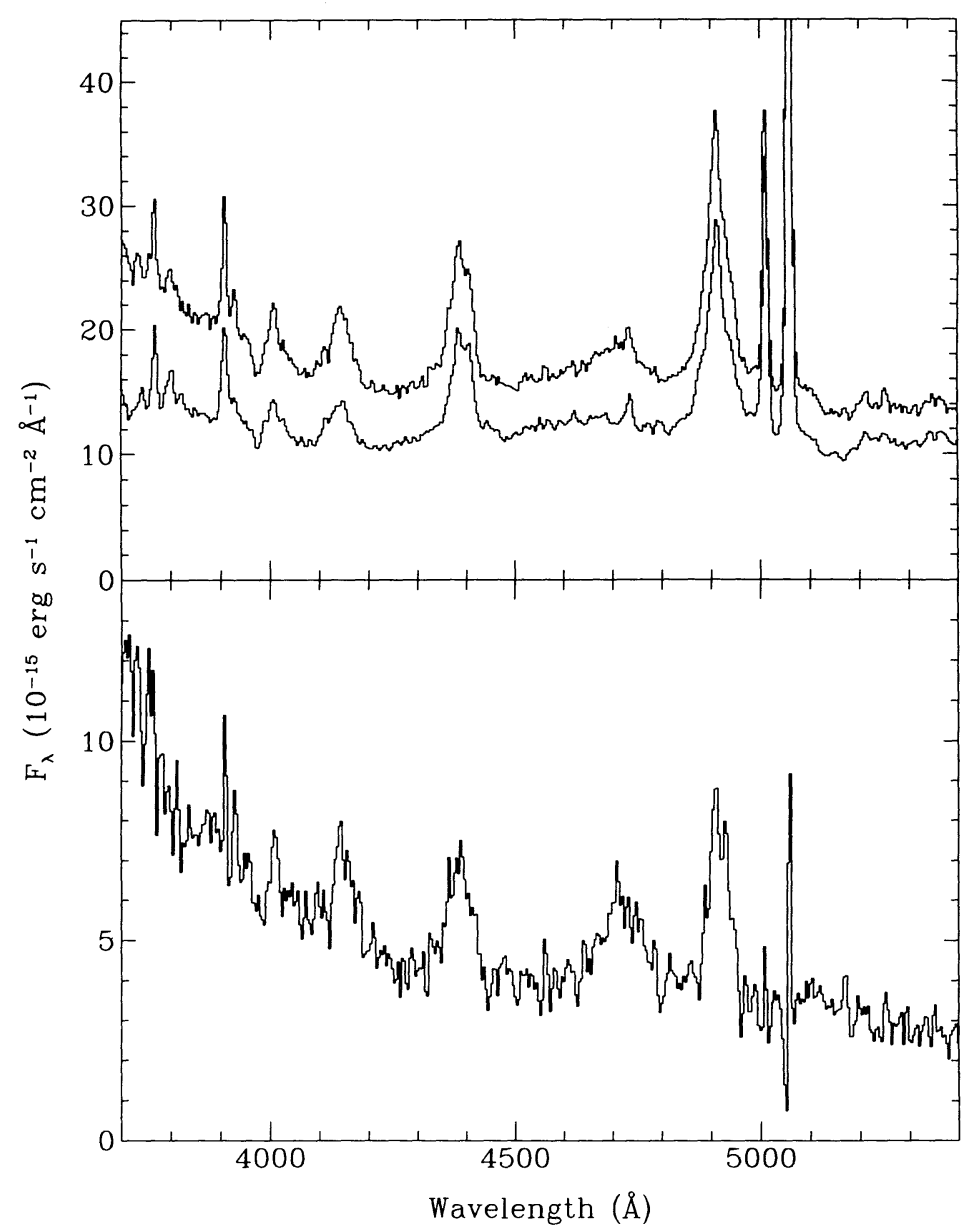

FIG. 6.-Top panel: the spectra obtained at CTIO on JD 2,448,660 (higher curve) and JD 2,448,704 (lower curve). Bottom panel: difference between the two spectra shown in the top panel. Notice how the continuum and all the main broad emission lines (particularly He II $\lambda 4686$ ) underwent a strong decrease.

VBO (Tables 2-4) shows that the spectrum is harder when the source is more luminous, confirming the trend displayed by the $I U E$ data. This is due at least in part to dilution by the stellar component. Stronger variability has been observed in the past: the $U$ flux varied by 0.38 mag at most during this campaign, while differences in excess of $1.1 \mathrm{mag}$ are present in the $U$ light curve shown by Glass (1992). The variation amplitude of $\mathbf{H} \beta$ is lower than that of most UV lines, and comparable to that of $C$ Iv 21549 . If a constant of $7 \times 10^{-14} \mathrm{erg} \mathrm{s}^{-1} \mathrm{~cm}^{-2}$ is subtracted from all $\mathrm{H} \beta$ fluxes, in order to correct for the contribution of the narrow component, $R_{\max }$ increases slightly to $1.4 \pm 0.1$.

As already mentioned, a comparison between the light curves in Figure 4 and those presented in Paper $V$ reveals strong similarities. As well as the deep, broad minimum observed in the UV continuum between JD 2,448,680 and JD $2,448,720$, most of the secondary minima also have counterparts in the optical curves: those at JD 2,448,631, JD 2,448,668, and JD 2,448,739 are visible in the continuum curve, and those at JD 2,448,668, JD 2,448,728, JD 2,448,739, JD 2,448,767, and JD $2,448,819$ can be observed, with a delay, in the light curve of $\mathrm{H} \beta$. Notice that in the $\mathrm{H} \beta$ curve the minimum at JD 2,448,767 (delayed to $\sim$ JD $2,448,775$ ) is of depth comparable to that of the main minimum, while it is much less pronounced in the UV continuum curves: the two minima have comparable depths also in the light curve of C III] $\lambda 1909$ (Paper V).
Unfortunately, most of the period during which the $I U E$ was observing at intervals of 2 days instead of 4 days (JD 2,448,783 to JD $2,448,833$ ) was disturbed by bad weather at CTIO and ESO, leading to large gaps and poorer quality in the optical light curves. Thus, while we cannot exclude that the shorttimescale "flickering" observed in the UV curves (Paper V) is present also in the optical light curves, the evidence for this is weak.

Figure 7 shows the cross-correlation functions (CCFs) of the optical continuum and $\mathrm{H} \beta$ light curves with the $F_{1460}$ continuum curve presented in Paper V. The CCFs were calculated with the method described by Gaskell \& Peterson (1987), without artificially extending the light curves beyond their first and last points (in other words, for each lag only the overlapping branches of the curves were cross-correlated). The discrete cross-correlation functions (DCFs) were also calculated as described by Edelson \& Krolik (1988), with the differences introduced in Paper V. As Figure 7 shows, the CCFs and DCFs are in good agreement. Notice that each CCF was obtained from light curves which were derived from completely independent data sets: there is therefore no influence on the CCFs by correlated errors as when line and continuum fluxes are obtained from the same spectra (Edelson \& Krolik 1988).

The window autocorrelation function (ACF) for the optical continuum (also shown in Fig. 7) was obtained by averaging the ACFs of many white-noise light curves sampled with the 


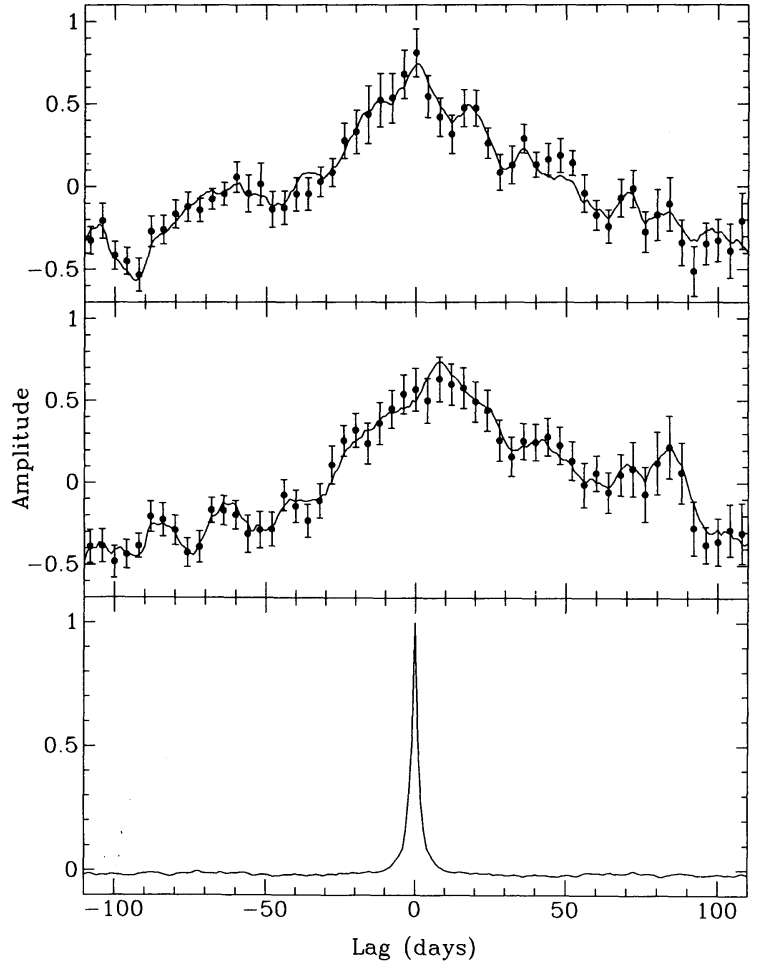

Fig. 7.-Top panel: the CCF (continuous line) and DCF (filled circles) of the $F_{1460}$ continuum curve (Paper V) vs. the optical continuum curve shown in Fig. 4. The DCF is calculated in 4 day bins. Middle panel: the CCF and DCF of the $F_{1460}$ continuum curve vs. the $\mathrm{H} \beta$ curve shown in Fig. 4. Bottom panel: the window ACF for the sampling pattern of the optical continuum. The window ACF for the sampling of the $\mathrm{H} \beta$ curve is slightly broader (FWHM $=2.7$ days instead of 2.1 days).

same temporal pattern as the continuum curve (Gaskell \& Peterson 1987). The full width at half-maximum (FWHM) of the sampling window ACF is 2.2 days, which indicates how much artificial correlation is introduced by interpolating the light curve in order to calculate the CCF. The window ACF for the $\mathrm{H} \beta$ light curve is slightly broader ( $F W H M=2.7$ days). Both window ACFs are much narrower than the CCFs, indicating that the variability on timescales larger than about 2.5 days has been resolved.

The results of the CCF analysis are summarized in Table 10: for each CCF, $\Delta t$ (peak) is the lag at which it reaches the maximum value, $\Delta t$ (center) is the average of the two halfmaximum lags, and $r_{\max }$ is the peak value. Cross-correlating $\mathrm{H} \beta$ and the optical continuum with any of the three continuum light curves presented in Paper $\mathrm{V}$ yields virtually identical results. How to obtain a reliable uncertainty for the peak lag is still a controversial matter: the method indicated by Gaskell \& Peterson (1987) gives an uncertainty of 2 days for all the values of $\Delta t$ (peak) listed in Table 10. The true uncertainty may be somewhat higher than this but probably does not exceed 4 days.

Notice that the FWHMs of the CCFs are considerably larger than those of the CCFs presented in Paper V. One reason for this is the lower ratio between the amplitude and noise of the variations in the optical light curves. Another possible reason is their less regular sampling. In particular, if the CCFs are calculated without including the points after JD $2,448,777$ (thus eliminating the influence of the largest gap in the sampling pattern), the FWHMs of the CCFs become 22.8
TABLE 10

Cross-Correlation Results

\begin{tabular}{|c|c|c|c|c|}
\hline Feature & $\begin{array}{c}\Delta t \text { (peak) } \\
\text { (days) }\end{array}$ & $\begin{array}{c}\Delta t \text { (center) } \\
\text { (days) }\end{array}$ & $r_{\max }$ & $\begin{array}{c}\text { FWHM } \\
\text { (days) }\end{array}$ \\
\hline Optical contin & 1 & 1.6 & 0.741 & 41.9 \\
\hline Optical continuum versus $F_{1835} \ldots$ & 0 & 1.3 & 0.746 & 42.2 \\
\hline Optical continuum versus $F_{2700} \ldots$ & 0 & 2.1 & 0.760 & 44.4 \\
\hline$F(\mathrm{H} \beta)$ versus $F_{1460} \cdots$ & 8 & 7.2 & 0.737 & 40.0 \\
\hline$F(\mathrm{H} \beta)$ versus $F_{1835}$ & 8 & 7.4 & 0.742 & 39.9 \\
\hline$F(\mathrm{H} \beta)$ versus $F_{2700}$ & 9 & 19.6 & 0.684 & 65.2 \\
\hline
\end{tabular}

days for the optical continuum and 35.3 days for $\mathrm{H} \beta$. Moreover, the main minimum is broader in the $\mathrm{H} \beta$ curve than in the continuum curves, and this will naturally broaden the CCF of $\mathrm{H} \beta$. Paper V presented evidence that the variability of NGC 3783 may not be statistically stationary: the irregular sampling of the ground-based campaign could therefore strongly influence the shape of the CCF. The position of the CCF peak, however, is stable. The very large FWHM of the $\mathrm{H} \beta$ versus $F_{2700} \mathrm{CCF}$ is caused by the fact that its peak is low enough to bring a broad plateau at positive lags just above the halfmaximum level.

The optical versus UV continuum CCF reaches its maximum at 0-1 days: as for NGC 5548 (Paper II), therefore, there is no strong evidence that the optical continuum is delayed with respect to the UV continuum. The lag between the light curves of $\mathrm{H} \beta$ and $F_{1460}$, as derived from the peak of the CCF, is $\sim 8$ days. This is a factor of 2.5 less than the equivalent result for NGC 5548, and confirms the difference in line versus continuum lags between the two objects, which was evidenced by the IUE data (Paper V). Isolating the main minimum in the optical and $F_{1460}$ curves and repeating the cross-correlation yields peak lags of 1 and 6 days for the optical continuum and $\mathrm{H} \beta$, respectively. The last minimum observed in the $\mathrm{H} \beta$ curve between JD 2,448,820 and JD $2,448,844$, when isolated, yields a lag of 8 days with respect to the corresponding feature of the UV continuum. The difference between lags obtained from isolated minima or from the entire light curves is not significant within the uncertainties discussed above.

Because the near-IR light curves do not show any obvious similarity to the UV and optical curves, we have not used them in the cross-correlation analysis. Glass (1992) found that the near-IR flux followed variations in the optical continuum $(U$ band) at an interval of 80-90 days. While the UV and optical data do not exactly precede the IR by showing a steplike change at the appropriate place, the dip around JD 2,448,700 is followed by an increase which precedes the IR step by about this amount. However, it is unlikely that the two features are related: if they were, one would have to explain the lack of a similar decrease in the IR flux before the brightening and the fact that the rise in flux occurs on comparable timescales (15-20 days) while the emitting regions must be of considerably different sizes.

\section{SUMMARY AND CONCLUSIONS}

We have presented the results obtained from the groundbased monitoring of the Seyfert 1 galaxy NGC 3783, conducted in 1991-1992 in parallel with the IUE monitoring campaign described in Paper V. Light curves of the optical continuum and of $\mathrm{H} \beta$, sampled at median intervals of 2.0 and 2.1 days, respectively, were obtained from spectroscopic and 
photometric observations obtained at several observatories. The main results confirm the similarities and differences between NGC 3783 and NGC 5548 evidenced by the IUE data, and can be summarized as follows:

1. The optical continuum underwent significant variability, and its light curve strongly resembles that of the UV continuum, with lower amplitude variations.

2. Cross-correlation analysis reveals no observable delay between the variations of the optical and UV continua, within the (possibly conservative) uncertainty of 4 days. This extends to the optical band the trend displayed by the UV continuum light curves (Paper V).

3. A significant increase occurred in the near-IR flux at the end of the campaign, but no feature in the UV/optical continuum curves is an obvious "driver" for it. Considering the width of the $K$ versus $U$ CCF in Glass (1992), it is likely that the UV/optical continuum variations were not intense or long enough for corresponding features, if any are present, to be visible in the near-IR curves.

4. The light curve of $\mathbf{H} \beta$ also strongly resembles those of the continua. Cross-correlation analysis reveals that its lag with respect to the UV continuum is about 8 days. As in NGC 5548
(Papers I-III and VII), the light curve of $\mathrm{H} \beta$ in NGC 3783 is more delayed with respect to the continuum light curve than those of the high-ionization lines. The lag of $\mathrm{H} \beta$ with respect to the continuum is at least twice as long as that of $\mathrm{Ly} \alpha$, about twice that of C IV, and comparable to that of $\mathrm{Mg}$ II $\lambda 2798$.

5. The $\mathrm{H} \beta$ versus continuum lag in NGC 3783 is shorter than in NGC 5548, just as the lags of the high-ionization lines in NGC 3783 are shorter than their counterparts in NGC 5548 (Paper V).

The authors are grateful to all the observatories involved for the generous allocation of observing time, and to J. Baldwin, N. Brosch, M. Goad, E. J. A. Meurs, H. Netzer, E. Pérez, E. Rokaki, J. Roland, and W. Wamsteker, who supported the proposals but did not feel that their contribution was sufficient for them to be coauthors. The Porto Alegre CCD camera, used for the observations at CASLEO, is operated under a contract between UFRGS (Brazil) and CASLEO (Argentina). C. W. acknowledges a fellowship granted by the Brazilian Institution CNPq. This work was partly supported by NSF grant AST9117086 and NASA grant NAG5-1824 (both to Ohio State University).
Alloin, D., et al. 1994, in preparation

Carter, B. S. 1990, MNRAS, 242,

Clavel, J., et al. 1991, ApJ, 366, 64 (Paper I)

Clavel, J., Wamsteker, W., \& Glass, I. S. 1989, ApJ, 337, 236

de Ruiter, H. R., \& Lub, J. 1986, A\&AS, 63, 59

Dietrich, M., et al. 1993, ApJ, 408, 416 (Paper IV)

Edelson, R. A., \& Krolik, J. H. 1988, ApJ, 333, 646

Evans, I. N. 1988, ApJS, 67, 373 1989, ApJ, 338, 128

Gaskeli, C. M., \& Peterson, B. M. 1987, ApJS, 65, 1

Glass, I. S. 1992, MNRAS, 256, 23P

Hayes, D. S., \& Latham, D. W. 1975, ApJ, 197, 593

Landolt, A. U. 1973, AJ, 78, 959

. 1983, AJ, 88, 439

Maoz, D., et al. 1993, ApJ, 404, 576

Menzies, J. W., \& Feast, M. W. 1983, MNRAS, 203, 1P

\section{REFERENCES}

Osmer, P., Smith, M., \& Weedman, D. W. 1974, ApJ, 189, 187

Pelat, D., Alloin, D. \& Fosbury, R. A. E. 1981, MNRAS, 195, 787

Peterson, B. M. 1993, PASP, 105, 247

Peterson, B. M., et al. 1991, ApJ, 368, 119 (Paper II)

Peterson, B. M., et al. 1992, ApJ, 392, 470 (Paper III)

Peterson, B. M., et al. 1994, ApJ, 425, 622 (Paper VII)

Reichert, G. A., et al. 1994, ApJ, 425, 582 (Paper V)

Stirpe, G. M., de Bruyn, A. G., \& van Groningen, E. 1988, A\&A, 200, 9

van Groningen, E., \& Wanders, I. 1992, PASP, 104, 700

Winge, C., Pastoriza, M. G., \& Storchi-Bergmann, T. 1990, Rev. Mexicana Astron. Af., 21, 177

Winge, C., Pastoriza, M. G., Storchi-Bergmann, T., \& Lipari, S. 1992, ApJ, 393, 98

Winkler, H. 1992, MNRAS, 257, 677

Winkler, H., Glass, I. S., van Wijk, F., Marang, F., Spencer Jones, J. H., Buckley, D. A. H., \& Sekiguchi, K. 1992, MNRAS, 257, 659 